\title{
A importância do marketing e da administração para consultórios odontológicos
}

\author{
The importance of marketing and administration for dental offices \\ La importancia del marketing y la administración para los consultorios dentales
}

Maria Aparecida Lucena de Oliveira Motta ORCID: https://orcid.org/0000-0002-6109-6910 Centro Universitário Maurício de Nassau, Brasil E-mail: mariaparecida@gmail.com

Herrison Félix Valeriano da Silva ORCID: https://orcid.org/0000-0001-6714-3151 Universidade Federal da Paraíba, Brasil E-mail: Herrison.felix.vds@gmail.com Rafaella Bastos Leite ORCID: https://orcid.org/0000-0002-3304-120X Centro Universitário Maurício de Nassau, Brasil E-mail: rrafaella_bastos@hotmail.com Jussara da Silva Barbosa ORCID: https://orcid.org/0000-0001-6123-5266 Faculdade Nova Esperança, Brasil E-mail: Barbosajsara@gmail.com Juan Vitor Costa Leite ORCID: https://orcid.org/0000-0002-6069-6703 Faculdade Nova Esperança, Brasil E-mail: juan.leit@hotmail.com

Carolyne Matias de Oliveira Felismino ORCID: https://orcid.org/0000-0002-2344-8356 Faculdade Nova Esperança, Brasil E-mail: carolyne-matias@hotmail.com

Maria Eduarda de Araujo Cruz ORCID: https://orcid.org/0000-0001-8876-8249 Faculdade Nova Esperança, Brasil

E-mail: eduardaaraujo207@gmail.com

Gabriella Pires de Gusmão ORCID: https://orcid.org/0000-0001-7634-7076 Faculdade Nova Esperança, Brasil E-mail: gabriellapg12@gmail.com

Laíse Nascimento Correia Lima ORCID: https://orcid.org/0000-0002-1773-847X Universidade Federal da Paraíba, Brasil E-mail: laiselima@msn.com

Rayanne Hellen do Nascimento Silva ORCID: https://orcid.org/0000-0003-2541-7470 Centro Universitário Maurício de Nassau, Brasil E-mail: rayanne@gmail.com

\begin{abstract}
Resumo
O presente trabalho tem como objetivo debater como o marketing (que ganhou muita importância no meio digital, nos últimos anos) pode melhorar a imagem da empresa, quando bem feito e respeitando a ética odontológica, e explanar sobre gestão de clínicas odontológicas - visto que organização de documentos, respeito aos cronogramas e controle de custos acarreta em maior excelência, além de diminuir gastos e amortecer riscos -, encontrando assim soluções com a finalidade de dinamizar o desempenho dos estabelecimentos. Trata-se de uma revisão da literatura, na qual foi realizada uma busca eletrônica de publicações na base de dados PubMed, Lilacs e Scielo, utilizando-se as seguintes palavras-chave, obtidas de acordo com o Medical Subject Headings (MeSH): odontologia, marketing e gestão, com o operador booleano "AND"; sendo selecionados 7 trabalhos. Conclui-se que é essencial que os cursos de graduação em odontologia adotem modelos mais efetivos sobre o assunto não técnico para a formação de novos profissionais, o que melhorará a qualidade de serviços prestados e possibilitará maiores chances de sucesso profissional e financeiro da empresa.
\end{abstract}

Palavras-chave: Odontologia; Marketing; Gestão. 


\begin{abstract}
This paper aims to discuss how marketing (which has gained a lot of importance in the digital environment in recent years) can improve the company's image, when done well and respecting dental ethics, and explaining about the management of dental clinics - since organization of documents, respect for schedules and cost control leads to greater excellence, in addition to reducing expenses and mitigating risks -, thus finding solutions with the purpose of boosting the performance of establishments. This is a literature review, where an electronic search for publications was carried out in the PubMed, Lilacs and Scielo database, using the following keywords, obtained according to the Medical SubjectHeadings (MeSH): dentistry, marketing and management, with the Boolean operator "AND"; 7 papers were selected. It is concluded that it is essential that undergraduate courses in dentistry adopt more effective models on the non-technical subject for the training of new professionals, which will improve the quality of services provided and will enable greater chances of professional and financial success for the company.
\end{abstract}

Keywords: Dentistry; Marketing; Management.

\title{
Resumen
}

Este artículo tiene como objetivo discutir cómo el marketing (que ha ganado mucha importancia en el entorno digital en los últimos años) puede mejorar la imagen de la empresa, cuando se hace bien y respetando la ética dental, y explicar la gestión de las clínicas dentales, desde la organización de los documentos. , el respeto por los horarios y el control de costos conduce a una mayor excelencia, además de reducir gastos y mitigar riesgos, encontrando así soluciones con el propósito de impulsar el desempeño de los establecimientos. Se trata de una revisión de la literatura, donde se realizó una búsqueda electrónica de publicaciones en la base de datos PubMed, Lilacs y Scielo, utilizando las siguientes palabras clave, obtenidas según los Medical SubjectHeadings (MeSH): odontología, marketing y gestión, con el operador booleano". Y"; Se seleccionaron 7 artículos. Se concluye que es fundamental que los cursos de pregrado en odontología adopten modelos más efectivos en el tema no técnico para la formación de nuevos profesionales, lo que mejorará la calidad de los servicios. proporcionados y permitirán mayores posibilidades de éxito profesional y financiero para la empresa.

Palabras clave: Odontología; Márketing; Administración.

\section{Introdução}

O crescimento do número de novos profissionais e o aumento da atuação das empresas de convênios na área odontológica influenciaram substancialmente na estrutura do mercado, ocasionando a necessidade do uso de conceitos de administração para aperfeiçoar a gestão da empresa, logo, um bom resultado do consultório odontológico está associado a dois fatores essenciais: planejamento estratégico de qualidade e capacidade em gestão administrativa (Costa et al., 2015).

De acordo com Tomaz (2011), o cirurgião-dentista precisa ter conhecimento das técnicas não clínicas, como relacionamento pessoal, administração e marketing. Nas faculdades de odontologia do Brasil, pouco se aborda sobre gerenciamento e não existem, na maioria das instituições de ensino, disciplinas específicas voltadas para o mercado de trabalho que contenham informações e conhecimentos básicos para administração e gestão de consultório; contudo, este também é um modelo de negócio. É através da gestão que o dentista planeja compras, organiza atendimentos, especifica serviços e se torna capaz de identificar riscos e vícios dentro do estabelecimento, melhorando assim a qualidade de seus tratamentos.

Da mesma forma, há pouco conhecimento e certo receio em se falar de marketing na área da saúde. Investir no marketing já foi considerado uma "mercantilização" da profissão e trazia incertezas sobre os aspectos éticos e legais da prática. Atualmente temos uma visão diferenciada, em especial do marketing odontológico: gradativamente percebemos que ele pode ser benéfico tanto para o dentista quanto para o paciente (S.I.N. Implant System, 2019).

Para Zuchini e colaboradores (2012), o marketing odontológico tem como objetivo criar um bom relacionamento entre o consumidor e o cirurgião-dentista, além de propiciar a conquista de novos clientes e a obtenção de uma melhor atuação no mercado de trabalho, fornecendo as condições necessárias dentro de um cenário tão competitivo como o atual. O marketing também é responsável pela criação do vínculo emocional entre o dentista e o cliente, fazendo este enxergar além dos produtos e serviços oferecidos e transformando a relação entre consultório e paciente em uma combinação saudável, bilateral e transparente.

Segundo Paranhos (2011, p. 221), 
"Marketing na prática odontológica pode ser considerado o processo de chamar pacientes ao consultório sem ferir o código de ética da profissão. A maneira pelo qual o cirurgião-dentista faz marketing determina em grande parte, o sucesso da clínica. Nos dias atuais várias são as dificuldades encontradas pelo profissional para atrair e, mais ainda, fidelizar seus clientes. Compete ao marketing estabelecer com eles uma relação duradoura de troca, oferecendo-lhes soluções capazes de satisfazer necessidades e expectativas".

O marketing odontológico é um espaço que pode ser dividido em: marketing tradicional, marketing digital, marketing interno, marketing externo e marketing de relacionamento, sendo imprescindível que o cirurgião-dentista conheça um pouco sobre cada um deles, de modo que consiga colocá-los em prática. O sucesso surge de uma soma de fatores: qualidade dos serviços prestados, trabalho em um ambiente limpo e confortável, boa relação entre profissional e cliente, segurança e organização (Medeiros; Lima 2001 apud Chuva; Lacerda, 2019).

A alta concorrência também acaba por impor a necessidade de buscar novos nichos e é nesse cenário que começam a surgir clínicas odontológicas com foco em públicos específicos (Bini, 2020). Antigamente o único mecanismo de marketing de que os cirurgiões-dentistas precisavam era uma boa reputação, mas os tempos são outros e o marketing se transformou em uma ferramenta flexível que, com dedicação, poderá levar seu trabalho rumo ao sucesso (Paranhos et al., 2010).

Dadas todas as circunstâncias apresentadas acima e a atual situação da odontologia no Brasil, como já descrita, Ribas et al. (2010) entende que se faz necessário a prática de uma gestão de qualidade e um marketing eficaz dentro de um consultório odontológico. Aquilo que o dentista considerava desnecessário para o seu desempenho profissional se mostrará, a partir do nosso tempo, cada vez mais essencial para a sua sobrevivência no mercado de trabalho.

O propósito deste trabalho é explanar sobre a importância da gestão e do marketing de consultórios odontológicos, e o emprego dessas práticas (e até mesmo a associação da palavra empresa com consultório odontológico) provoca repulsa aos ouvidos de muitos dentistas sempre que são pronunciadas, não sendo aceitas ideias para elaboração de projetos administrativos ou de propaganda. A gestão é de grande importância para avaliar resultados obtidos através do marketing e também está relacionada com a capacidade de levantar situações financeiras como custos e faturamento. Este trabalho tem como finalidade desmistificar o tema e trazer à luz métodos que podem melhorar o desempenho destes estabelecimentos, inclusive em qualidade de atendimento e tratamento.

\section{Metodologia}

Trata-se de uma revisão da literatura, fazendo uma pesquisa qualitativa, na qual foi realizada uma busca eletrônica de publicações na base de dados PubMed, Lilacs e Scielo, utilizando-se das seguintes palavras-chave, obtidas de acordo com o Medical Subject Headings (MeSH): odontologia, marketing e gestão, com o operador booleano "AND”; foram selecionados 11 trabalhos (Koche,2011).

Adotou-se os seguintes critérios de inclusão para a busca dos estudos:

- Ter sido divulgado no período de janeiro de 2015 a janeiro de 2020;

- Estudos que avaliaram o marketing e a gestão como ferramentas de ajuda na imagem e organização da empresa;

- Estudos que correlacionavam os problemas de administração e propaganda de consultórios odontológicos.

Foram adotados os seguintes critérios de exclusão:

- Artigos de qualquer outra língua que não fosse à portuguesa;

- Artigos não relacionados ao tema, além de trabalhos de conclusão de cursos, tese e dissertações.

Ao final, após a aplicação dos critérios de eleição para a seleção dos manuscritos para o desenvolvimento do estudo, foram excluídos 04 artigos que não se enquadravam nos critérios pré-estabelecidos, sendo, portanto, incluídos 07 artigos de maior relevância sobre o tema e utilizados para a realização desse trabalho. 
Este trabalho trata de expor questões sobre administração e propaganda para dentistas. A abordagem utilizada foi do tipo qualitativa, na tentativa de encontrar soluções que possam ser aplicadas corretamente, aumentando a lucratividade e melhorando a qualidade do atendimento e do serviço prestado. Esta pesquisa também tem a finalidade de dissertar sobre como aperfeiçoar o desempenho dos estabelecimentos odontológicos diante de uma competitividade acirrada e como o marketing pode ser capaz de posicionar/melhorar a imagem da empresa.

\section{Resultados e Discussão}

Sabe-se que o uso das técnicas de marketing é aceitável no ramo odontológico, mas de forma que não haja desvalorização da profissão. Com isso, o Código de Ética Odontológico estabeleceu regras relacionadas às práticas publicitárias, com o intuito de constituir padrões para um bom desenvolvimento da profissão (Soares, 2019, apud Felício et al., 2013; Martins et al., 2011).

Nota-se que atualmente há uma crescente no número de consultórios odontológicos, diante disso, observa-se um cenário empresarial competitivo e que muitas vezes fica refém de incertezas e tantas turbulências, fazendo com que os cirurgiões-dentistas busquem cada vez mais ferramentas e técnicas para que estas auxiliem no processo gerencial (Costa, 2015).

$\mathrm{Na}$ Tabela 1 que se segue, encontram-se informações de 07 artigos que demonstram os objetivos e resultados selecionados para esta revisão, além de dados referentes a autor, tema, ano, local e páginas dos respectivos artigos.

Tabela 1. Título, país e ano de publicação, objetivos e resultados dos artigos analisados no estudo. $\mathrm{n}=7$.

\begin{tabular}{|c|c|c|c|c|}
\hline AUTORES & TÍTULO & $\begin{array}{c}\text { PAÍS } \\
\text { E } \\
\text { ANO }\end{array}$ & OBJETIVO(S) & RESULTADO(S) \\
\hline $\begin{array}{c}\text { GARBIN, Cléa et } \\
\text { al. }\end{array}$ & $\begin{array}{l}\text { O uso das redes sociais } \\
\text { naodontologia: uma } \\
\text { análise dos aspectos } \\
\text { éticos de páginas de } \\
\text { clínicas odontológicas }\end{array}$ & $\begin{array}{c}\text { Brasil } \\
2018\end{array}$ & $\begin{array}{l}\text { Argumentar sobre a força que tem } \\
\text { ganhado as redes sociais como } \\
\text { ferramentas de comunicação, } \\
\text { publicidade e propaganda e } \\
\text { explorar dados sobre o uso pelos } \\
\text { dentistas de acordo, ou não, com o } \\
\text { Código de ÉticaOdontológico. }\end{array}$ & $\begin{array}{l}\text { Constatou-se que, do total de } 102 \text { das } \\
\text { páginas de clínicas odontológicas } \\
\text { analisadas, } 76,5 \% \text { não continha algumitem } \\
\text { obrigatório. Também foi identificado } \\
\text { oferecimento de serviços gratuitos, } \\
\text { anúncios de modalidades de pagamentos, } \\
\text { prêmios ou descontos. Obs.: foi } \\
\text { desconsiderada informação } \\
\text { sobre "antes e depois", permitido pelo } \\
\text { CFO recentemente. }\end{array}$ \\
\hline $\begin{array}{c}\text { SOARES, } \\
\text { Karolyne } \\
\text { de Melo et al. }\end{array}$ & $\begin{array}{l}\text { Descumprimento do } \\
\text { Código de Ética } \\
\text { Odontológica em redes } \\
\text { sociais-análise de } \\
\text { irregularidades } \\
\text { vinculadas à publicidade e } \\
\text { propaganda }\end{array}$ & $\begin{array}{c}\text { Brasil } \\
2019\end{array}$ & $\begin{array}{l}\text { Alertar sobre os padrões exigidos } \\
\text { pelo CEO quanto à publicidade e } \\
\text { propaganda, tendo maior } \\
\text { concentração de infrações as } \\
\text { estratégias de marketing } \\
\text { encontradas na internet. }\end{array}$ & $\begin{array}{l}\text { Um total de } 76 \% \text { dos } 133 \text { perfis } \\
\text { pesquisados não exibe número de inscrição } \\
\text { do CRO (essa porcentagem é de } 98 \% \\
\text { quando se trata de perfis de } \\
\text { pessoas jurídicas). } 14 \% \text { dos avaliados } \\
\text { exibiam tanto preços quanto formas de } \\
\text { pagamento. Usuários não habilitados } \\
\text { realizam revenda e promoções de } \\
\text { produtos odontológicos sem prescrição } \\
\text { profissional, desvalorizando o dentista e } \\
\text { colocando em risco a saúde dos usuários. }\end{array}$ \\
\hline
\end{tabular}




\begin{tabular}{|c|c|c|c|c|}
\hline $\begin{array}{l}\text { JÚNIOR, Ernani } \\
\text { de Souza } \\
\text { Guimarães et } \\
\text { al. }\end{array}$ & $\begin{array}{c}\text { Marketing em } \\
\text { Serviços Odontológicos: } \\
\text { Práticas e Resultados a } \\
\text { partir da Percepção do } \\
\text { Profissionais }\end{array}$ & $\begin{array}{l}\text { Brasil } \\
2015\end{array}$ & $\begin{array}{l}\begin{array}{l}\text { Entrevista de } 30 \text { profissionais de } \\
\text { diferentes }\end{array} \text { estabelecimentos } \\
\text { odontológicos e verificação } \\
\text { quanto à utilização de marketing. }\end{array}$ & $\begin{array}{l}\text { OMS recomenda } 01 \text { dentista a cada } \\
1.500 \text { habitantes; na cidade onde se } \\
\text { desenvolveu o estudo há } 01 \text { a cada } 440 \\
\text { habitantes, o que sugere mercado de alta } \\
\text { competição. } 80 \% \text { dos dentistas percebem o } \\
\text { "boca a boca" como a mais influente; } \\
13,3 \% \text { apontam encaminhamentos de } \\
\text { outros profissionais também como muito } \\
\text { importantes; propagandas em jornais } \\
\text { impressos locais e rádios são apontadas } \\
\text { como capaz de atrair clientes por } 33.3 \% \\
\text { (embora } 90 \% \text { critiquem os jornais por } \\
\text { não atingir o público-alvo e não reverter } \\
\text { nos benefícios desejados). } 100 \% \text { das } \\
\text { clínicas populares usam panfletagem } \\
\text { (também são as que mais investem em } \\
\text { propaganda). 26.7\% não fazem qualquer } \\
\text { uso de publicidade. } 46.7 \% \text { dão } \\
\text { descontos durante negociação. } 33.3 \% \\
\text { realizam contato com o cliente após } \\
\text { prestação de serviços (sendo todos } \\
\text { especialistas em prótese, o que pode } \\
\text { configurar necessidade e não estratégia } \\
\text { de marketing). } 53.3 \% \text { apresentam o desejo } \\
\text { de aumentar número de clientes. }\end{array}$ \\
\hline
\end{tabular}

Fonte: Dados da pesquisa (2021).

Após análise realizada dos artigos evidenciados neste trabalho, observa-se que ainda encontramos muitas clínicas e consultórios odontológicos que não se enquadram dentro das premissas do Código de Ética Odontológico, uma vez que anúncios irregulares (com preços, formas de pagamento e gratuidade, dentre outras situações) ainda é prática comum. Também se constata um elevado número de estabelecimentos que não exibem a inscrição de seus respectivos CROs e/ou responsável técnico, seja em suas fachadas, seja no meio virtual.

Garbin e colaboradores (2018, p.27) afirmam:

[...] foi identificado oferecimento de brindes, serviços gratuitos, anúncios de modalidades de pagamento, bonificação entre outros favorecimentos que são proibidos segundo o Código de Ética Odontológico.

Reforçando o exposto acima, Soares e colaboradores (2019) identificam 76\% de clínicas odontológicas que não exibem números de inscrição de conselho regional odontológico e $14 \%$ de estabelecimento que mostram preços e formas de pagamentos, erroneamente.

Independente de opiniões pessoais e, considerando que a odontologia possui conselho de classe e código de ética, as práticas irregulares devem ser combatidas. É de responsabilidade e empenho dos gestores (dentistas ou não) terem conhecimento do que trata as normas da profissão e enquadrar suas ações dentro daquilo que é permitido, agindo com respeito e criatividade no que compete ao marketing e propaganda. A Resolução 196/2019 do Conselho Federal de Odontologia, embora estabeleça possibilidades outrora proibidas, exige em todas as publicações de vídeos e imagens o nome do profissional e o número de inscrição; não fazendo, a própria norma, outras distinções, entende-se da necessidade de incluir tais informações em todos os posts de redes sociais que se conectam com a profissão (sejam eles provenientes de anúncios pagos ou não); as páginas iniciais de redes sociais também devem conter as informações aqui explicitadas.

Face ao exposto, ações imprescindíveis devem ser realizadas por parte dos órgãos e instituições responsáveis pelas fiscalizações, a fim de reduzir as infrações já existentes (Soares et al., 2019).

Ainda em relação à divulgação e marketing, algo ainda comum é identificar clínicas e consultórios odontológicos realizando ações como panfletagens ( $\mathrm{O}$ que é vedado pelo CRO), em cruzamento de ruas ou lugares com grande fluxo de 
pessoas, como em centros comerciais, próximo a estações de ônibus ou outro ponto que se entenda estratégico, sendo tal trabalho considerado importante para a maioria das clínicas que se autodenominam populares; também é corriqueiro ver propagandas destes estabelecimentos em jornais, revistas, rádio ou até mesmo televisão (Que são permitidas, desde que sigam as normas).

Claro que toda ação de marketing é válida e estas citadas são complementares uma das outras; ocorre que tais ações são também dispendiosas, exigem planejamento estratégico e financeiro em um nível que muitas vezes empresas de pequeno e médio porte não são capazes de suportar, realizando apenas ações pontuais, sem dar continuidade, por falta de dinheiro e sem compreender, ao final das contas, se tal estratégia obteve êxito ou não, pois não há uma forma precisa de identificar se o público foi atingido.

A não identificação do retorno da campanha e o gasto vultoso passam a desencorajar o dentista a continuar realizando tais campanhas de marketing, mesmo mantendo uma boa gestão e controle de informações oriundas das ações de propaganda, com a recepção sempre realizando perguntas aos clientes, tais como “como você nos conheceu?", ainda assim é possível que a pessoa não dê uma resposta exata ou que a recepcionista se esqueça de realizá-la de vez em quando. Os dados obtidos não parecem robustos e o retorno, por fim, se mostra pequeno. As informações sobre os resultados que se pode obter com esse tipo de marketing quase sempre vem de seus vendedores, é comum o representante de uma rádio, por exemplo, apresentar valores de campanhas e resultados que podem ser alcançados; quando não se atinge essa expectativa ou não se tem a percepção dela, o dentista se frustra com a campanha.

É nesse contexto que o uso das redes sociais tem ganhado força como estratégia de comunicação, uma vez que 102 milhões de brasileiros têm acesso à internet, pois a comunicação por meio desta é fácil e pode ser visualizada por qualquer usuário, na maioria das vezes. Desta forma, a realização da propaganda fica facilitada e, ao utiliza- lá, o cirurgião-dentista pode alcançar um número de pessoas com o menor investimento possível. É comum os consultórios odontológicos terem páginas nas redes sociais e as mesmas devem estar de acordo com as normas dispostas no Código de Ética Odontológica (Garbin et al., 2018).

Para Soares et al. (2019, p. 2):

As redes sociais têm sido utilizadas como ferramentas para atingir o público-alvo desejado, com o objetivo de divulgação dos serviços e produtos. O uso da internet tem se mostrado uma estratégia simples, barata, eficaz e amplamente utilizada para propaganda.

De acordo com Garbin e et al. (2018, p. 23):

O avanço tecnológico e a popularização do uso da internet favorecem o uso das mídias sociais para divulgação de serviços e estabelecimentos como forma de angariar novos clientes. Desta forma a odontologia também utiliza desses meios, porém, nem sempre eles estão de acordo com as normas do Código de Ética Odontológico, que nos diz respeito ao anúncio, a propaganda e publicidade.

É no marketing virtual que as empresas mais apostam atualmente, Facebook, Instagram, Google Adwords, LinkedIn, Twitter e Pinterest estão entre os aplicativos mais utilizados e possibilitam ao consultório ficar "no ar" vinte e quatro horas por dia, possuem ferramentas para respostas automáticas em caso de dúvidas comuns e possibilitam que o cliente entre em contato diretamente, além de entregar localização de horários de funcionamento de forma direta e simples.

Tais ferramentas possibilitam a interação e a verificação de possíveis interessados que seguem a página e visitam os conteúdos postados, um olhar atento às mídias em redes sociais, com manutenção regular dos perfis e conteúdo, a interação nestes e também em sites de internet, aliado a anúncios virtuais e publicidade tradicional realizados de forma estratégica, a fim de atingir um público definido (adolescentes e pessoas mais novas para manutenções ortodônticas, idosos e pessoas mais 
velhas para próteses e implantes, por exemplo) e de acordo com a capacidade financeira do consultório, tem tudo para ter um retorno interessante ao estabelecimento e garantir a satisfação do dentista com os resultados obtidos.

Uma das coisas que se deve ter em vista é que não é de qualquer forma que se investe em marketing, é necessário atribuir uma parcela do faturamento para tal fim. Um número razoável e aceito entre as empresas de pequeno porte é o emprego de 5\% do total faturado no mês, contudo, em campanhas onde se percebe um retorno maior, pode- se investir até $10 \%$ em marketing e propaganda.

Outro desafio, que também se relaciona com os princípios básicos para o bom funcionamento dos estabelecimentos de saúde, é a gestão, que requer a utilização de ferramentas como indicadores e softwares capazes de controlar informações de pacientes (o que resulta em maior clareza e fidedignidade) e também dados como manutenções de equipamentos, contas a pagar e receber, agendamentos, dedetização entre outros.

Segundo Limeira e colaboradores. (2018, p. 162):

A gestão em saúde pode ser reconhecida como a direção ou condução de processos político-institucionais relacionados ao sistema de saúde, desenvolvendo-se nos níveis técnico-administrativo e técnico-operacional. Gerir organizações de saúde requer competências específicas traduzidas em seus conhecimentos, habilidades e atitudes, capacidade de análise situacional, tomada de decisões, apresentação de soluções, resolução de problemas e administração de conflitos. Por isto se faz necessário que as Instituições de Ensino Superior (IES) acompanhem essas mudanças vivenciadas no setor da saúde e as incorporem em suas bases de ensino.

Sales e colaboradores (2015) explicam que o conhecimento nas ferramentas certas para gestão do consultório ou clínica odontológica ajudará o cirurgião-dentista a tomar as decisões certas, uma vez que a maioria desses estabelecimentos são administrados pelos próprios dentistas, com pouco conhecimento de gestão e planejamento, pontos importantes para o resultado financeiro.

Conforme artigos relacionados neste trabalho, é possível verificar que disciplinas de gestão e administração de clínicas ainda são pouco exploradas em cursos superiores de odontologia, sendo dada a prioridade para assuntos teóricos e técnicos do ofício e atribuindo pouca ou nenhuma importância às noções básicas que um dentista deve possuir para organização de sua própria clínica.

A habilidade de administrar é uma tendência imprescindível para o sucesso da clínica odontológica, porém poucos cirurgiões-dentistas tiveram oportunidades de acesso ao assunto na graduação, visto isso, entende-se a necessidade no aprendizado de gestão para fortalecer a classe e aperfeiçoar a qualidade dos serviços odontológicos.

Vale salientar que as empresas na área odontológica têm que atentar aos devidos cuidados quanto à organização documental, atualizando-os sempre que possível para uma futura necessidade, pois estes servem de prova e de anteparo para a tomada de decisão oportuna.

Desafio ainda maior é para recém-formados em se inserir no mercado de trabalho, pois além da pouca experiência técnica, também não possuem clientela cativa e experimentam pouco conhecimento de administração.

Há três alternativas para cirurgiões-dentistas: trabalhar na saúde pública, em consultórios privados de terceiros ou arriscar-se em um consultório próprio. Caso o profissional decida realizar sua carreira em serviço próprio, necessitará entender sobre organização e orientação estratégica. É crescente o número de consultórios odontológicos que, diante da complexidade no quadro empresarial e de tantas dificuldades e incertezas, estão buscando ferramentas e técnicas para que estas auxiliem na dinâmica gerencial (Costa et. al., 2015). 


\section{Conclusão}

A partir do estudo realizado conclui-se que, para um adequado desempenho de um consultório odontológico é necessário manter o bom funcionamento de um “tripé” que envolve, além do conhecimento técnico do próprio profissional, sua boa relação com o marketing odontológico e com a gestão da empresa em que atua. Apesar da necessidade de conhecimento das técnicas não clínicas - como relacionamento pessoal, administração e marketing -, o cirurgião-dentista tem pouco ou nenhum contato com tais assuntos durante sua formação; conforme artigos relacionados neste trabalho, é possível verificar que disciplinas de gestão e administração de clínicas ainda são pouco exploradas em cursos superiores de odontologia, sendo dada a prioridade para assuntos teóricos e técnicos.

Após revisão da literatura pôde-se concluir que com a competitividade no mercado odontológico, se faz necessário utilizar-se do marketing interno e do marketing externo. Os cirurgiões dentistas, fazendo uso destes recursos de maneira ética e adequada, tenderão a conseguir resultados positivos no que compete à captação de novos pacientes, bem como na fidelização dos antigos, além de melhorar a imagem do consultório. Tanto os cirurgiães dentistas, quanto os consultórios odontológicos, devem respeitar as normas de comunicação e divulgação na odontologia.

As ferramentas de administração, como os softwares para gestão, vêm sendo desenvolvidas pelo ramo da tecnologia da informação, trazendo diariamente novidades que permitem aos profissionais da área odontológica gerenciar suas atividades, auxiliando efetivamente na tomada de decisões com indicadores robustos, eliminando, assim, tarefas desnecessárias e repetitivas, deve-se conhecer, administrar e controlar com muita propriedade os custos e as despesas gerados através da comercialização de serviços.

Após análise dos artigos pôde-se concluir que é de suma importância para o cirurgião-dentista lançar mão do conhecimento da gestão e do marketing, uma vez que os mesmos direcionam o estabelecimento para uma atuação mais profissional e de maior qualidade. É o conhecimento dessas áreas que também proporcionam ao dentista quantificar ganhos (lucro, faturamento, aumento ou diminuição de pacientes, ticket médio, entre outros), traçar metas e analisar indicadores para tomadas de decisões mais assertivas, diminuindo riscos operacionais e aumentando significativamente as chances de prosperar dentro de um mercado atualmente tão competitivo.

Como as regras e estratégias de marketing, evoluem ao longo dos anos, os autores sugerem, estudos periódicos sobre o marketing na odontologia, para sempre atualizar a literatura, sobre tal tema, que está em constante mudança e evolução.

\section{Referências}

Aguilar, R. (2017). Como apresentar o preço e fechar o orçamento? - Parte 6. Blog Dental Cremer. https://blog.dentalcremer.com.br/como-o-fecharorçamento.

Amaral, B. (2020) 16 ferramentas que todo profissional deveria conhecer. Smlbrasil. https://blog.smlbrasil.com.br/3-ferramentas-que- todo-analista-deprocesso-deveria-conhecer.

Bini, D. (2020). SEO Local para dentistas: posicionamento é determinante para ter sucesso. SEOHacks. https://www.seohacks.com.br/blog/seo- local-paradentistas-posicionamento-e-determinante-para-ter-sucesso.

Bolina, L. (2020). O que são gatilhos mentais e como utilizá-los na sua estratégia de Marketing! Rockcontent. https://rockcontent.com/br/blog/g atilhosmentais/\#: :text=Gatilhos\%20mentais\%20s\%C3\%A3o\%20est\%C3\%ADmulos\%20recebidos,resultados\%20para\%20marketing\%20e\%20vendas.

Capez, F. (2020). Venda casada: entenda o que é e os direitos do consumidor. Brasil econômico. https://economia.ig.com.br/colunas/defesa-doconsumidor/2020-06-19/venda-casada-entenda-o-que-e-e-os-direitos-do- consumidor.html.

Carneiro, W. (2019). A gestão odontológica é uma característica empreendedora. Odontobranding. Recuperado de https://odontobranding.com.br/a-gestaoodontologica-e-uma-caracteristica-empreendedora.

Carreon, T. (2016). Master book of administration: Marketing em Odontologia. Napoleão.

Cassita, D. (2020). Software de gestão de processos: 5 ferramentas para sua empresa.Caetreinamentos. https://caetreinamentos.com.br/blog/gestaoempresarial/software-gestao-processos. 
Código De Ética Odontológica. (2012) Conselho Regional de odontologia de São Paulo. ResoluçãoCFOn ${ }^{\circ} 118-2012$. http://www.crosp.org.br/uploads/etica/6ac4d2e1ab8cf02b189238519d74fd45.pdf.

Conselho Federal De Odontologia. (2019). Código de ética odontológico. Resolução CFO n 196/2019.

Costa, R. M., Ribeiro, N. C. R., Tunes, U. R., Nunes, M., Rocha D. \& Lago, M. S. (2015). Odontoclínica: simulação de gestão em clínica odontológica em um curso de graduação em odontologia. Revista Abeno, 15(1), 77-85.

Chuva, R. R. \& Lacerda, R. P. (2019). Análise da influência do marketing odontológico para o cirurgiãodentista. 38f. TCC (Graduação)- Curso de Odontologia- Faculdade de Odontologia de Porto Nacional- Faculdade Presidente Antônio Carlos, Porto Nacional, 2019

Dib, L. (2017). História da Odontologia. Luciano Dib odontologia estomatologia. https://lucianodib.com.br/historia.php.

Gabriel, B. (2020). Como realizar um pós-venda que dá certo? Orgânico natural marketing. https://www.organicadigital.com/blog/pos-venda-que- da-certo.

Garbin, C. A. S., Amaral, M. A., Garbin, A. J. I. \& Saliba, T. A. (2018). Análise lexical do Código de Ética Odontológica, Revista de odontologia da UNESP, 47(2), 79-84.

Garbin, C. A. S., Ortega, M. M., Garbin, A. J. I. \& Saliba, T. A. (2018). O uso das redes sociais na odontologia: uma análise dos aspectos éticos de páginas de clínicas odontológicas, Revista Brasileira de odontologia, 5(1), 22-29.

Iwakura, F. (2020). 10 dicas para aumentar o fechamento de contratos. Consultor de Dentistas. https://consultordedentistas.com.br/2016/08/21/10- dicas-paraaumentar-o-fechamento-de-contratos.

Koche, J. C. (2011). Fundamentos de metodologia científica. Petrópolis: Vozes. http://www.brunovivas.com/wpcontent/uploads/sites/10/2018/07/K\%C3\%B6che-Jos\%C3\%A9-Carlos0D0AFundamentos-de-metodologia-cient\%C3\%ADfica-_teoria-da0D0Aci \%C3\%AAncia-e-inicia\%C3\%A7\%C3\%A3o-\%C3\%A0 pesquisa.pdfhttps://repositorio.ufsm.br/bitstream/handle/1/15824/Lic_Computacao_MetodologiaPesquisa-Cientifica.pdf?sequence $=1$

Limeira, F. I. R., Rebouças, P. R. M., Rocha, E. A. L. S. S \& Catão, M. H. C. V. (2018). O ensino de gestão nos cursos de graduação em Odontologia no Brasil. Revista Abeno, 18(1), 161-19.

Lolli, L. F., Silva, M. C., Campos, F. N. L., Mathias, A. P \& Nemer, M. R. M. (2019). Odontologia defensiva e educação permanente: gestão de prontuários contribuindo na formação de cirurgiões-dentistas com responsabilidade profissional. Revista Abeno, 19(2), $113-122$.

Medeiros, H. O. (2019). Auditoria Hospitalar. Editora Telesapiens, 43p.

Merlone, T. (2020). A importância da administração de custos. Portal Educação. https://www.portaleducacao.com.br/conteudo/artigos/pedagogia/aimportancia-da- administracao-de-custos/14174.

Ministério da Saúde. (2020). o que é covid-19. Gov.br. https://coronavirus.saude.gov.br/sobre-a-doenca.

Miranda, S. S., Bulcão, J. A \& Dultra, C. A. (2015). Publicidade em odontologia: Avaliação dos aspectos éticos envolvidos. Revista Brasileira de Odontologia Legal- RBOL. 2(1), 53-67.

Paranhos, L. R.; Benedicto, E. N.; Fernandes, M. M.; Viotto, F. R. S \& Daruge JR, E. (2011). Implicações éticas e legais do marketing na Odontologia. Revista RSBO. 8(2), 219-24.

Passos, G. (2020). Dentistas poderão acompanhar pacientes por meio virtual durante a pandemia. AgênciaBrasil. https://agenciabrasil.ebc.com.br/radioagencia-nacional/acervo/saude/audio/2020- 06/dentistas-poderao-acompanhar-pacientes-distancia-durante-pandemia.

Peçanha, V. (2015). Marketing Digital: O que é isso, afinal? http://marketingdeconteudo.com/marketing-digital.

Natali, F. A. (2010). Marketing em odontologia. 33f. TCC (Graduação) - Curso de Odontologia - Faculdade de odontologia de Araçatuba - Universidade Estadual Paulista "Júlio de Mesquita Filho", Araçatuba.

Ribas, M. A., Siqueira, E. S., \& Binotto, E. (2010). O Desafio da Gestão para profissionais da Odontologia. XXX Encontro nacional de engenharia de produção. São Carlos- SP.

Ribeiro, A. (2019). Procon-ES e CRO-Es notificam lojas por venda casada e comercialização irregular de planos odontológicos. Procon, Espírito Santo. https://procon.es.gov.br/Not\%C3\%ADcia/procon-es-e-cro-es- notificam-lojas-por-venda-casada-e-comercializacao-irregular-de-planos- odontologicos.

Rocha, H. (2013). Gatilhos Mentais: o que são e 10 exemplos do uso no marketing. Clickpages. https://klickpages.com.br/blog/gatilhos-mentais.

Soares, K. M., Rolim, A. K. A., Silva, D. F. B., Silva, C. A. M., Campos, F. A. T., Silva, M. W., Costa, M. R. M \& Santos, C. A. O. (2019). Descumprimento do código de Ética Odontológica em redes sociais- análise de irregularidades vinculadas à publicidade e propaganda, Revista eletrônica acervo saúde, v.11, n. 16 , p. 1-7.

Souza, J. \& Ostermann, A.C. (2015). Controlando o incontrolável: A aplicação das regras de atendimento na construção da compreensão mútua entre clientes e atendentes em um call Center. Linguagem em (Dis)curso- LemD,.15(1), 13-32.

Silveira, S. M \& Moura, M. A. (2010). Scripts de atendimento em call centers; uma visão de documentos eletrônicos. Enc. Bibli: R. Eletr. Bibliotecon. Ci. Inf., 15(29), 145-168.

Spyere, P. R. (2013). Odontologia, ética e legislação. Museus das profissões: odontologia. http://museudasprofissoes.blogspot.com/p/historia- da-odontologiano-brasil.html. 
Research, Society and Development, v. 10, n. 6, e49210615858, 2021

(CC BY 4.0) | ISSN 2525-3409 | DOI: http://dx.doi.org/10.33448/rsd-v10i6.15858

Salves, W., Vallim, C. R., Barcellos, S. S \& FIORIN, I. (2015). Gestão de consultórios e clínicas odontológicas na cidade de Vitória: um mapeamento para entender as necessidades dos gestores. XII congresso Brasileiro de Custos-Foz de Iguaçu.

Silva, N. (2013). Os objetivos e os tipos de gestão de custos. Administradores.com, 2013. Recuperado de https://administradores.com.br/artigos/os-objetivose-os-tipos- da-gestao-de-custos.

Tomaz, P. A. R. (2011). Marketing para Dentistas: Orientações ao consultório- Empresa. (5a ed.).

ZuchinI, A. R. B., Lolli, M. C. G. S., Lolli, L. F. \& Lolli, H. A. (2012). Perfil profissional do cirurgião dentista em associação ao conhecimento e utilização de marketing. Arquivo odontológico, 43(1), 19-25. 